\title{
Relationship between residual viable myocardium and LV remodeling post-MI: Only part of the story
}

\author{
Robert J. Gropler, $\mathrm{MD}^{\mathrm{a}}$ \\ ${ }^{a}$ Cardiovascular Imaging Laboratory, Division of Radiological Sciences, Mallinckrodt Institute of \\ Radiology, Washington University School of Medicine, St. Louis, Missouri
}

Received Jan 23, 2016; accepted Jan 24, 2016

doi: $10.1007 / \mathrm{s} 12350-016-0425-3$

\section{See related article, pp. 657-667}

Acute coronary syndrome, which accounts more than 1 million hospital admissions annually, reflects the assemblage of clinical symptoms secondary to acute myocardial ischemia that manifests as unstable angina or myocardial infarction (MI): either nonST elevation MI or ST elevation myocardial infarction (STEMI). ${ }^{1}$ Optimal initial therapy is timely and successful reperfusion to maximize myocardial preservation and salvage, with primary percutaneous coronary intervention (PCI) for STEMI being a prime example. This is followed by aggressive pharmacotherapy to preserve the gains of initial reperfusion therapy and the prevention of subsequent adverse left ventricular (LV) remodeling. In cases of MI where reperfusion therapy has not been instituted or is incomplete, a key question is whether the presence of resting mechanical dysfunction displayed by myocardium subtended by the infarct-related artery reflects predominantly scar or viable tissue (e.g., myocardial stunning). An accurate distinction appears useful for assigning prognosis and guiding further revascularization procedures. $^{2}$

Measurements of myocardial glucose metabolism with PET and ${ }^{18}$ F-fluorodeoxyglucose (FDG) and myocardial scar with MRI and delayed gadolinium contrast enhancement (DCE) are generally accepted as the two most accurate approaches for detecting viable

Reprint requests: Eliana Reyes, MD, Cardiovascular Imaging Laboratory, Mallinckrodt Institute of Radiology, 510 S. Kingshighway, St. Louis, MO, 63110; Groplerr@mir.wustl.edu J Nucl Cardiol 2017;24:668-71.

1071-3581/\$34.00

Copyright (C) 2016 American Society of Nuclear Cardiology. myocardium. However, the accuracy of these techniques, while high in patients with LV dysfunction due to chronic $\mathrm{CAD}$, diminishes significantly in patients evaluated early ( $\sim$ 1-2 weeks) following a MI, independent of the institution of reperfusion therapy. ${ }^{3,4}$ In the case of PET with FDG, marked variability in myocardial glucose metabolism in the early post-MI interval that is exacerbated by the presence reperfusion and the occurrence of post-inflammatory conditions that result in the myocardial accumulation of glycolytic proinflammatory cells are the likely contributors to the reduced accuracy of this method. ${ }^{5,6}$ It is well established that MRI with DCE overestimates the extent of myocardial scar in the early post-MI interval likely due to the effects of myocardial edema. ${ }^{3}$

The perfusable tissue index (PTI) is another approach for detecting myocardial viability. This index reflects the fraction of the myocardium that is capable of rapidly exchanging water, or said another way that is perfusable by water. It is posited that viable myocardium can exchange water, whereas myocardial scar or nonviable myocardium cannot. ${ }^{7}$ The PTI represents the water-perfusable tissue fraction $(\mathrm{PTF}, \mathrm{g} / \mathrm{ml}$ ) defined as the fraction of the water-perfusable tissue divided by the anatomic tissue fraction (ATF) within a given volume of region of interest. All of the necessary data to calculate PTI can be obtained from a PET/CT with ${ }^{15} \mathrm{O}$-water. In addition to measurement of myocardial blood flow (MBF), measurements of PTF, and the arterial and venous blood volumes are obtained from the ${ }^{15} \mathrm{O}$-water data acquisition. The ATF is determined from the CT attenuation image corrected for the arterial and venous blood volumes measured from the ${ }^{15} \mathrm{O}$-water data. ${ }^{8}$ Thus, a PTI value close to unity would be expected for a region of interest containing normal myocardium with a progressively decreasing value as the amount of nonviable myocardium or scar increases. Because PTI references the amount perfusable tissue relative to a total 
myocardium, it is also indicative of the total amount of viable myocardium (or conversely scar) within a myocardial segment exhibiting mechanical dysfunction. The results of numerous studies have demonstrated the accuracy of the method for detecting viable myocardium in patients with LV dysfunction due to chronic coronary disease. $^{9-11}$ Limited available data would suggest PTI may be equally accurate in patients early after MI and thus provide an advantage over PET with FDG and DCE measured with MRI in this patient population. ${ }^{7}$

In this edition of the Journal, Teunissen and colleagues compared the accuracy of PTI with MRI-derived DCE for identifying viable myocardium in patients with STEMI treated with successful PCI. Although in this patient population, the detection of viable is not of critical importance since the infarct-related artery is already revascularized, it permits assessment of these imaging techniques to identify viable myocardium (e.g., recovery of systolic function) without the confounding effects of intermittent or persistent ischemia. The patients consisted of primarily nondiabetic males who presented with their first STEMI and underwent successful revascularization (TIMI-3 flow in 95\% of patients) an average of $\sim 2$ hours after the onset of symptoms. At 4-6 days and $\sim 3$ mos post-MI, PET/CT with ${ }^{15} \mathrm{O}$-water and MRI with noncontrast and postcontrast methods was performed. The PET measurements included MBF and PTI, with the latter based on measurements of PTF and ATF. The MRI measurements included LV volumes, LV ejection fraction (LVEF), regional systolic wall thickening (SWT), DCE, and microvascular vascular injury (MVI). The latter likely reflects a no-reflow phenomenon due to a host of factors such as vasoconstriction, myocardial cellular edema, capillary endothelial cell swelling, and distal microembolization of atherosclerotic debris as well as hemorrhage and is a predictor of $\mathrm{LV}$ remodeling and poorer prognosis. ${ }^{12-14}$ The myocardium was divided into 16 segments within which measurements of MBF, PTI, DCE, and MVI were performed. These measurements were also extrapolated to provide a comparable measurement for the entire left ventricle. Myocardial segments were classified as remote from the site infarction (normal function without DCE) or dysfunctional (SWT $<3 \mathrm{~mm}$ ) measured on the baseline studies. Of those dysfunctional segments, $\sim 70 \%$ exhibited DCE and co-localized with the territory of the infarct-related artery. These segments were defined as infarcted myocardium with subgroups classified as viable or nonviable based on the presence or absence of SWT $\geq 3 \mathrm{~mm}$ at 3 mos, respectively.

Overall, PCI was successful as evidenced by average initial LVEF and LV volumes within the normal range that did not significantly change at follow-up.
Early post-MI, PTI of viable segments averaged 0.94 and was significantly higher compared to nonviable segments (0.80). This difference was consistent with the lower values for DCE and MVI (26\% and 29\% segmental extent, respectively) in viable compared with nonviable segments (59\% and $45 \%$, respectively). Of note, MBF did not differ between viable and nonviable segments. Based on area under the curve (AUC) analysis PTI and DCE were equivalent in predicting recovery of segmental function and both were superior to myocardial blood flow. Based on optimal cut-offs derived from AUC analysis, a PTI of $\geq 0.85$ exhibited a sensitivity, specificity, positive predictive value, and negative predictive value (NPV) of 85\%, 72\%, 67\%, and $88 \%$, respectively which compared favorably (although not statistically significant) with a DCE of $<32 \%$ which exhibited values of $72 \%, 69 \%, 58 \%$, and $80 \%$, respectively. With respect to global function at baseline, PTI $\geq$ 0.85 , infarct size by DCE, the presence and extent of MVI were all predictors of baseline LVEF. Based on multivariable analysis, the extent of MVI was the strongest and single independent predictor of baseline LVEF. In terms of recovery of global LV function, PTI was the only variable that significantly correlated with improvement of LVEF on univariable analysis.

Based on these results the authors correctly conclude the PTI method is an attractive approach for assessing myocardial viability in post-MI patients because of its high NPV, relatively simple and short image acquisition and processing and the very low radiation burden imposed by the study. Although requiring further study, the data suggest that PTI may be superior to DCE for predicting recovery of regional mechanical function in this patient population. As mentioned previously, viability detection is not typically needed for the patient population studied because they underwent successful primary PCI. However, it may be desirable in MI patients who present late after the initial event or where thrombolytic therapy is unsuccessful and subsequent revascularization is contemplated. Thus, techniques such as PTI would be of clinical utility. That being said, the advantages of PTI are significantly offset by the need for an on-site cyclotron and to a lesser extent the more limited availability of PET compared with SPECT, MRI, and echocardiography. Consequently, these disadvantages as well as the lack reimbursement for clinical ${ }^{15} \mathrm{O}$-water imaging in most countries, including the United States, make it unlikely the method will achieve more widespread clinical use.

However, the results of this study have made an important observation that is highlighted in Figure 4. That is, despite timely and complete recanalization of the infarct-related artery that resulted in at least $85 \%$ viable myocardium $(\mathrm{PTI} \geq 0.85)$ and the institution of 
standard post-MI pharmacotherapy, $\sim 25 \%$ of patients still exhibited a decline in LVEF at 3 mos. Moreover, many of these patients had a normal LVEF at baseline. Said another way, this subset of patients exhibited adverse LV remodeling. At first blush, the reader is left with question how can this be? It is generally understood LV remodeling post-MI typically occurs in those patients with reduced global function $(\mathrm{LVEF}<40)$ or an extensive myocardial scar, particularly in the anterior distribution. ${ }^{15}$ Indeed, it these patient groups in whom long-term inhibition of the renin-angiotensin system is recommended. However, these data are consistent with the results of prior studies. For example, in post-MI patients undergoing reperfusion therapy, $\sim 40 \%$ of dysfunctional myocardial segments without evidence of DCE failed to exhibit complete recovery of function. ${ }^{16}$ Taken in sum, these observations highlight the importance of other biological or mechanical determinants, beyond the extent of viable myocardium, on long-term LV function, and ultimately the prevention of adverse remodeling, even in cases where global LV function is initially preserved. Indeed, they are consistent with the known biology of post-MI LV remodeling which reflects perturbation in the key inflammatory and fibrotic components of myocardial repair. ${ }^{17}$ The challenge now is to develop the appropriate panel of biomarkers to accurately identify post-MI patients at risk for adverse LV remodeling. Although elevated plasma biomarkers such as measures of pro-inflammatory monocytes, matrix metalloproteinase activation, or apoptosis are associated with adverse outcome post-MI, they only identify a potentially high-risk patient. ${ }^{18-20}$ It is likely molecular imaging approaches will be needed to accurately localize and quantify the key biological processes which contribute to LV remodeling particularly those sited within the infarcted tissue and its border zone, the latter which represents viable myocardium. Furthermore, the extent to which these molecular processes work in synergy with structural and mechanical determinants of adverse remodeling will need to be determined. Once available, a more complete story of the potential for $\mathrm{LV}$ remodeling in post-MI patients can be told.

\section{Disclosure}

None

\section{References}

1. Krumholz HM, Keenan PS, Brush JE Jr, Bufalino VJ, Chernew ME, Epstein AJ, et al. Standards for measures used for public reporting of efficiency in health care: A scientific statement from the American Heart Association Interdisciplinary Council on Quality of Care and Outcomes research and the American College of Cardiology Foundation. J Am Coll Cardiol. 2008;52(18):1518-26.

2. van Loon RB, Veen G, Baur LH, Kamp O, Bronzwaer JG, Twisk $\mathrm{JW}$, et al. Improved clinical outcome after invasive management of patients with recent myocardial infarction and proven myocardial viability: Primary results of a randomized controlled trial (VIAMI-trial). Trials. 2012;13:1. Epub 2012/01/05. doi: 10.1186/1745-6215-13-1.

3. Dall'Armellina E, Karia N, Lindsay AC, Karamitsos TD, Ferreira $\mathrm{V}$, Robson MD, et al. Dynamic changes of edema and late gadolinium enhancement after acute myocardial infarction and their relationship to functional recovery and salvage index. Circ Cardiovasc Imaging. 2011;4(3):228-36.

4. Rubin PJ, Lee DS, Davila-Roman VG, Geltman EM, Schechtman $\mathrm{KB}$, Bergmann SR, et al. Superiority of C-11 acetate compared with F-18 fluorodeoxyglucose in predicting myocardial functional recovery by positron emission tomography in patients with acute myocardial infarction. Am J Cardiol. 1996;78(11):1230-5.

5. Buxton DB, Mody FV, Krivokapich J, Phelps ME, Schelbert HR. Quantitative assessment of prolonged metabolic abnormalities in reperfused canine myocardium. Circulation. 1992;85(5):1842-56.

6. Wollenweber TRP, Schäfer A, et al. Characterizing the inflammatory tissue response to acute myocardial infarction by clinical multimodality noninvasive imaging. Circ Cardiovasc Imaging. 2014;7:811-8.

7. Yamamoto $Y$, de Silva R, Rhodes CG, Araujo LI, Iida H, Rechavia E, et al. A new strategy for the assessment of viable myocardium and regional myocardial blood flow using 15O-water and dynamic positron emission tomography. Circulation. 1992;86(1):167-78.

8. Harms HJ, de Haan S, Knaapen P, Allaart CP, Lammertsma AA, Lubberink M. Parametric images of myocardial viability using a single 15O- $\mathrm{H}_{2} \mathrm{O}$ PET/CT scan. J Nucl Med. 2011;52(5):745-9.

9. de Silva R, Yamamoto Y, Rhodes CG, Iida H, Nihoyannopoulos P, Davies GJ, et al. Preoperative prediction of the outcome of coronary revascularization using positron emission tomography. Circulation. 1992;86(6):1738-42.

10. Iida H, Ruotsalainen U, Maki M, Haaparnata M, Bergman J, Voipio-Pulkki LM, et al. F-18 fluorodeoxyglucose uptake and water-perfusable tissue fraction in assessment of myocardial viability. Ann Nucl Med. 2012;26(8):644-55.

11. Knaapen P, Bondarenko O, Beek AM, Gotte MJ, Boellaard R, van der Weerdt AP, et al. Impact of scar on water-perfusable tissue index in chronic ischemic heart disease: Evaluation with PET and contrast-enhanced MRI. Mol Imaging Biol 2006;8(4):245-51. Epub 2006/06/23. doi: 10.1007/s11307-006-0044-5.

12. Cochet AA, Lorgis L, Lalande A, Zeller M, Beer JC, Walker PM, et al. Major prognostic impact of persistent microvascular obstruction as assessed by contrast-enhanced cardiac magnetic resonance in reperfused acute myocardial infarction. Eur Radiol. 2009;19(9):2117-26.

13. de Waha S, Desch S, Eitel I, Fuernau G, Zachrau J, Leuschner A, et al. Impact of early vs. late microvascular obstruction assessed by magnetic resonance imaging on long-term outcome after STelevation myocardial infarction: A comparison with traditional prognostic markers. Eur Heart J. 2010;31(21):2660-8.

14. Reffelmann T, Kloner RA. The "no-reflow" phenomenon: Basic science and clinical correlates. Heart. 2002;87(2):162-8.

15. O'Gara PT, Kushner FG, Ascheim DD, Casey DE Jr, Chung MK, de Lemos JA, et al. 2013 ACCF/AHA guideline for the management of ST-elevation myocardial infarction: A report of the American College of Cardiology Foundation/American Heart Association Task Force on Practice Guidelines. Circulation. 2013;127(4):e362-425. 
16. Beek AM, Kuhl HP, Bondarenko O, Twisk JW, Hofman MB, van Dockum WG, et al. Delayed contrast-enhanced magnetic resonance imaging for the prediction of regional functional improvement after acute myocardial infarction. J Am Coll Cardiol. 2003;42(5):895-901.

17. Frangogiannis NG. The inflammatory response in myocardial injury, repair, and remodelling. Nat Rev Cardiol. 2014;11(5):25565.

18. Kelly D, Khan SQ, Thompson M, Cockerill G, Ng LL, Samani N, et al. Plasma tissue inhibitor of metalloproteinase-1 and matrix metalloproteinase-9: Novel indicators of left ventricular remodelling and prognosis after acute myocardial infarction. Eur Heart J. 2008; 29(17):2116-24.

19. Maekawa Y, Anzai T, Yoshikawa T, Asakura Y, Takahashi T, Ishikawa $\mathrm{S}$, et al. Prognostic significance of peripheral monocytosis after reperfused acute myocardial infarction: A possible role for left ventricular remodeling. J Am Coll Cardiol. 2002;39(2): 241-6.

20. Soeki T, Tamura Y, Shinohara H, Sakabe K, Onose Y, Fukuda N. Relation between circulating soluble Fas ligand and subsequent ventricular remodelling following myocardial infarction. Heart. 2003;89(3):339-41. 